\title{
LV-EV-5
}

\section{Laparoscopic major hepatectomy using dorsal approach to the major hepatic veins}

\author{
Santiago LOPEZ BEN*
}

HPB Unit-General Surgery, Hospital Universitari de Girona Dr Josep Trueta, Girona, Spain

Lecture: Dorsal approach of right and middle hepatic veins lets their exposure from root to periphery, minimizing venous bleeding. Usefulness of this approach is shown to tradiitional major hepatectomies (right and left hemihepatectomies) as well as tecchnicaly major hepatectomies (anterior and posterior sectionectomies). 